\title{
Resonant photofragmentation of water at the oxygen $K$ edge by high-resolution ion-yield spectroscopy
}

\author{
M. N. Piancastelli, * A. Hempelmann, F. Heiser, O. Gessner, A. Rüdel, and U. Becker \\ Fritz-Haber-Institut der Max-Planck-Gesellschaft, 14195 Berlin, Germany
}

(Received 6 February 1998)

\begin{abstract}
Resonant photofragmentation of core-excited water molecules has been studied with high-resolution partialion-yield spectroscopy. Both the single and coincidence channels were monitored simultaneously. A steep increase in the coincidence yield curves above the ionization threshold, where the normal Auger decay produces doubly charged or multiply charged species that yield two or more charged fragments, has been observed. By comparing the single and coincidence channels the relative weight of $\mathrm{H}$ neutral fragments has been qualitatively assessed, and found to be higher for the $\mathrm{O} 1 s \rightarrow 4 a_{1}$ primary excitation. The presented ion-yield data provide strong evidence for a branching between atomic Auger decay and molecular fragmentation in the $\mathrm{O}^{+}$channel. The most unexpected result is the formation of $\mathrm{H}_{2}{ }^{+}$species being detected and attributed to a highly excited bending mode. [S1050-2947(99)00601-0]

PACS number(s): 33.80.Gj, 33.80.Eh
\end{abstract}

\section{INTRODUCTION}

The occurrence of dissociation following either core-tobound excitations below an inner-shell threshold or ionization above a core threshold in isolated molecules is a wellknown phenomenon [1] but the number of detailed studies dealing with this subject is still small compared to the analogous studies for condensed or chemisorbed molecules (i.e., the research area known as desorption induced by electronic transitions [2,3]). However, such observations are extremely useful in shedding light on both the character of the excited and ionized states, and on the dynamics of the photofragmentation processes. Different mechanisms can lead to photofragmentation above or below an ionization threshold. Above threshold, normal Auger decay produces two-hole states which may fragment easily. Below threshold, the highest probability of fragmentation pertains to the two-holeone-particle states (spectator line) reached after resonant $\mathrm{Au}$ ger (autoionization) decay. In this framework, it is assumed that the ion fragmentation is a subsequent step following electron decay, i.e., the time scale for electron emission is much faster than the nuclear separation. Exceptions exist when the molecular dissociation is ultrafast, i.e., it takes place on the same time scale as electron decay [4].

Ion-yield spectroscopy is particularly informative concerning the nature of fragmentation processes following molecular inner-shell excitation or photoionization, as it is possible to obtain the relative intensity of the ionic species in their final states. Different decay channels can exhibit very different behaviors, depending on the particular fragment or combination of fragments which are detected. In contrast to the many low- and intermediate-resolution studies in this field, the only isolated molecule studied with high-resolution ion-yield spectroscopy following synchrotron radiation excitation, is $\mathrm{CO}[5,6]$. In water, the photofragmentation has

\footnotetext{
*Permanent address: Department of Chemical Sciences and Technologies, University “'Tor Vergata,' 00133 Rome, Italy.
}

been investigated by ion-yield spectroscopy of condensed and chemisorbed layers [7]. In the gas phase, the dissociation of water has been studied by ion-yield spectroscopy following valence excitation [8]. In the core ionization region, the angular distribution of fragments [9] has been reported in low photon resolution angle-resolved ion-yield spectroscopy, but the yield of the different single channels was not measured separately. No high-resolution ion-yield complete experiments in the gas phase have been published as far as we know.

We report here a high-resolution study of photofragmentation of water in the photon energy region around the oxygen $K$ edge. Ion-yield spectra were obtained for single channels (ion yield of each fragment) and coincidence channels (two or three fragments detected in coincidence).

A steep increase is generally observed in the coincidence yield curves above the ionization threshold, where the normal Auger decay produces doubly charged or multiply charged species which yield two or more charged fragments. Below threshold, the fragment ion yield is still high, hinting at easily photofragmenting species. It has been observed that in water the spectator decay bears more weight than the participator decay even for resonant states with some antibonding molecular orbital character [10]. Therefore the high ion yield corresponds to the importance of spectator decay following the primary electron excitation.

This is particularly true for pure Rydberg states which decay predominantly through this decay mode into final twohole-one-electron states. These states have a stronger dissociative character than the participator final one-hole states of the decay of the mixed-Rydberg-antibonding molecular orbitals, thus exhibiting a fragmentation behavior analogous to doubly charged two-hole states reached by normal Auger decay.

Comparing single channels and coincidence channels, we can evaluate the relative weight of emission of neutral $\mathrm{H}$, which is the most important decay product of the $\mathrm{O} 1 s$ $\rightarrow 4 a_{1}$ resonance. As to the production of $\mathrm{O}^{+}$, a branching has been observed between atomic Auger decay and molecular fragmentation. A peculiar photofragmentation process in 


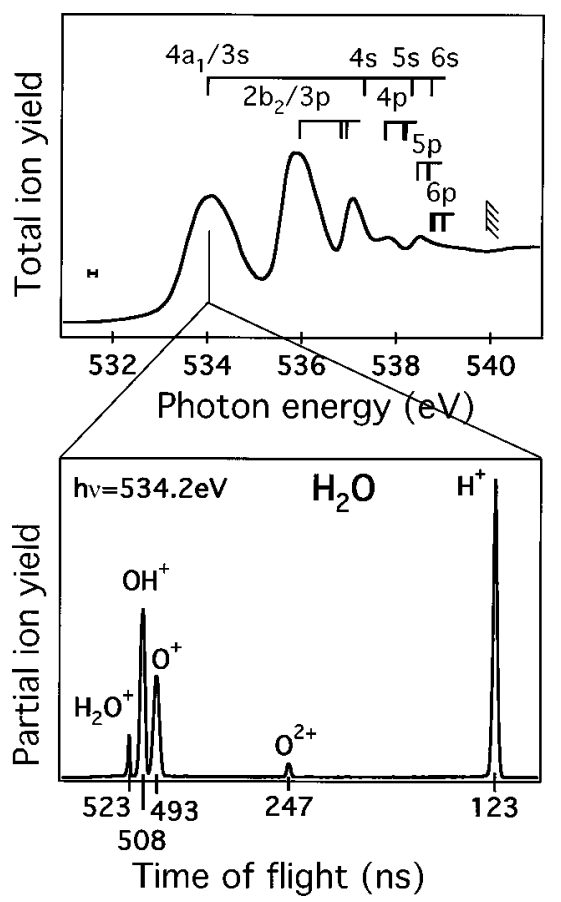

FIG. 1. Total ion yield near the $\mathrm{H}_{2} \mathrm{O}: \mathrm{O}(1 s)$ threshold. The lower part shows a time-of-flight mass spectrum taken at the first $\mathrm{H}_{2} \mathrm{O}: \mathrm{O}(1) \rightarrow 4 a_{1} / 3 s$ resonance. The energy resolution is marked in the lower left corner.

water is the formation of the $\mathrm{H}_{2}^{+}$species following the excitation to a core-to-bound state and the subsequent decay to a vibrationally highly excited final state. We propose a highly excited symmetric bending motion which can lead the two hydrogen atoms to bond-length-like distances as the origin for this direct formation of $\mathrm{H}_{2}^{+}$.

\section{EXPERIMENT}

The experiments were performed at beamline BW3 of the Hamburger Synchrotronstrahlungslabor. This beamline is a triple $N$-pole undulator beamline $(N=21,33$, and 44$)$ equipped with a high-resolution SX 700 plane-grating monochromator modified for high photon throughput. At the oxygen $K$-edge, we evaluated the photon bandwidth to be 170 $\mathrm{meV}$ with a photon flux of $10^{12}$ photons/s.

In order to record different partial ionization yields simultaneously during the photon energy scan, a newly developed time-of-flight mass spectrometer with a position-sensitive multihit detector was used. The spectrometer consists of a McLaren-type space-charge-focusing instrument, $42 \mathrm{~mm}$ long, with an extraction field of $900 \mathrm{~V} / \mathrm{cm}$, and a multihit anode. This arrangement makes it possible to measure all pairs of charged particles in coincidence, independent of their charge state and initial kinetic energy [11]. The capability to determine the direction of the ionic fragmentation was not used in the present study, because it would require much longer measuring time on one energy point than possible in a high-resolution energy scan with the photon flux, given in our experiment. The flight times of the ions were measured with respect to the bunch marker of the storage ring, which was operated in a double-bunch mode with a time window of $480 \mathrm{~ns}$, yielding intrinsic branching ratios of

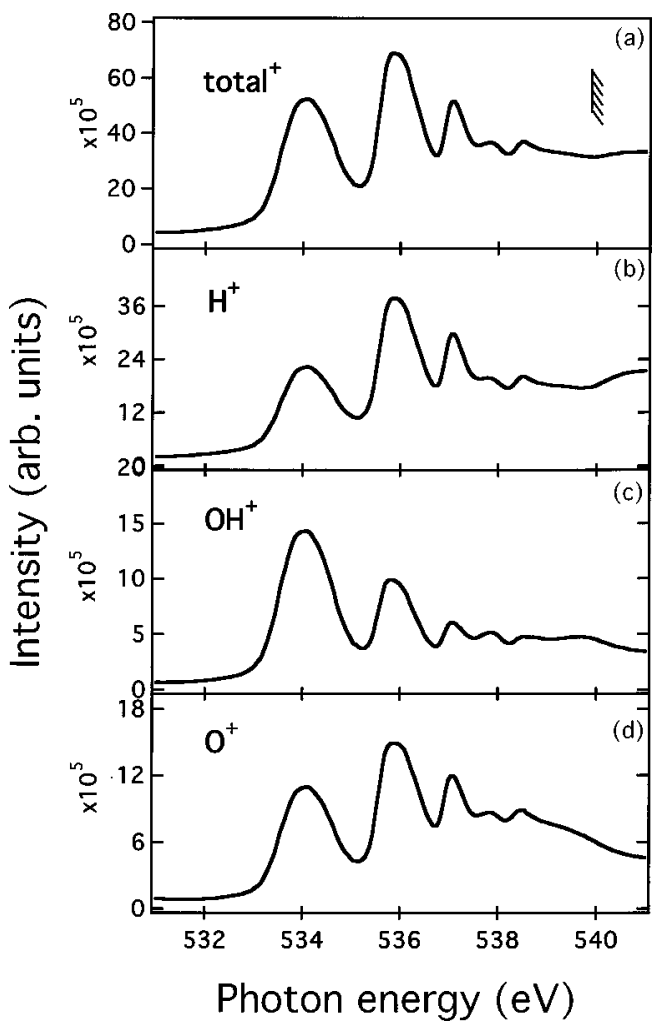

FIG. 2. Partial noncoincident ionization yields of $\mathrm{H}_{2} \mathrm{O}$ following $\mathrm{H}_{2} \mathrm{O}: \mathrm{O}(1 s)$ excitation: $\mathrm{H}^{+}(\mathrm{b}), \mathrm{OH}^{+}(\mathrm{c})$, and $\mathrm{O}^{+}(\mathrm{d})$ along with total ionization yield (a) on top of this figure.

fragment ions. The spectrometer was set at an angle of approximately $55^{\circ}$ with respect to the electric vector of the photon beam in order to reduce the effects of anisotropic fragmentation in the time-of-flight peaks which could have unwanted effects on the resolution of our energy scans [12]. The background pressure was better than $3 \times 10^{-6} \mathrm{~h} \mathrm{~Pa}$ during the measurements. In order to avoid possible distortions of the scans, all coincident and noncoincident ionization channels were measured simultaneously during one photon energy scan, including the monitoring of the background in the time-of-flight spectra. This capability is crucial for the comparison of photon-energy-dependent details in the different spectra. A more detailed description of this apparatus was presented elsewhere [11]. All scans are normalized with respect to the pressure and the photocurrent. Background subtraction has been performed for all coincident and noncoincident curves.

\section{RESULTS AND DISCUSSION}

Ion-yield spectra have been obtained in the $531-542-\mathrm{eV}$ photon energy range for single channels, double-coincidence channels, and triple-coincidence channels. Along with a mass spectrum taken on top of the first resonance, the totalion-yield spectrum is shown as the top curve of Fig. 1. It compares very well with the photoabsorption curve reported in the literature with analogous photon bandwidth [13]. This is not always the case, since the close correspondence between photoabsorption and ion yield implies that the majority of the final electronic states are equally dissociative and their ion yields approximately the same, which is evidently 


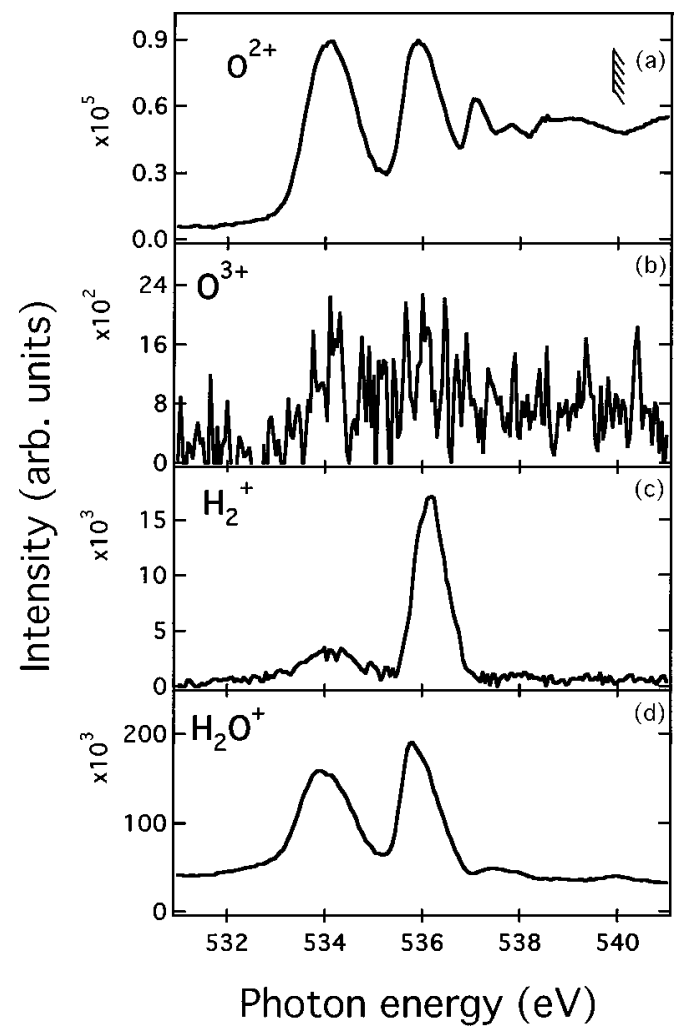

FIG. 3. Partial noncoincident ionization yields of $\mathrm{H}_{2} \mathrm{O}$ following $\mathrm{H}_{2} \mathrm{O}: \mathrm{O}(1 s)$ excitation: $\mathrm{O}^{2+}(\mathrm{a}), \mathrm{O}^{3+}(\mathrm{b}), \mathrm{H}_{2}^{+}$(c), and $\mathrm{H}_{2} \mathrm{O}^{+}$(d).

the case for water. We have used the assignments made in the analysis of photoabsorption measurement [13]. The first three intense peaks in the total ion yield, at 534.0, 535.9, and $537.1 \mathrm{eV}$, are related to the $\mathrm{O} 1 s \rightarrow 4 a_{1}, \mathrm{O} 1 s \rightarrow 2 b_{2}$, and $\mathrm{O}$ $1 s \rightarrow 3 p b_{1}+3 p a_{1}$ resonant processes, respectively. The first two are assigned to a transition from the ground state to a mixture of empty molecular orbitals and Rydberg states, while the third transition is of pure Rydberg character. The following more complex structures are assigned to several overlapping Rydberg states. The ionization threshold is located at $539.9 \mathrm{eV}$.

A close examination of the total-ion-yield features in Fig. 1 reveals the absence of a resolved vibrational structure which is expected to be approximately $150 \mathrm{meV}$ [14]. This could be either due to the lack of instrumental resolution $(\approx 170 \mathrm{meV})$ or to the nature of the core-excited states, which are known to be strongly dissociative, and therefore might not support vibrational substates. This lack of vibrational structure has been reported several times in literature concerning different experiments on both isolated and condensed water molecules [7].

We may observe some general features when comparing single (Figs. 2 and 3) and coincidence channels (Figs. 4 and 5). A common feature is the behavior above the ionization threshold at $539.9 \mathrm{eV}$. We can observe an enhancement of the coincidence channels compared to the single channels. This enhancement is similar in the triple-coincidence scans (Fig. 5). This may be regarded as a general effect, and attributed to the presence of normal Auger decay above the ionization limit which greatly enhances the production of doubly or multiply charged species. Therefore, the fragmentation processes which produce two or more charged species at

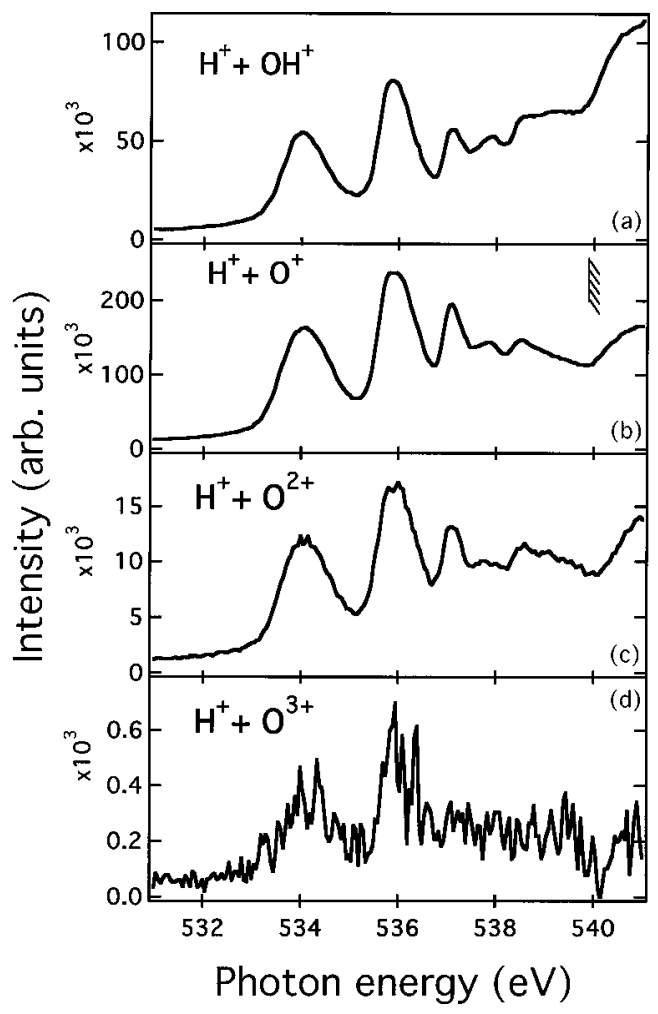

FIG. 4. Coincidence channels of the dissociative ionization of $\mathrm{H}_{2} \mathrm{O}$ following $\mathrm{H}_{2} \mathrm{O}: \mathrm{O}(1 s)$ excitation and ionization: $\mathrm{H}^{+}+\mathrm{OH}^{+}$(a), $\mathrm{H}^{+}+\mathrm{O}^{+}(\mathrm{b}), \mathrm{H}^{+}+\mathrm{O}^{2+}(\mathrm{c})$, and $\mathrm{H}^{+}+\mathrm{O}^{3+}$ (d).

once are more likely following normal Auger decay with a doubly charged ion as the final state than resonant Auger decay which produces mostly highly excited singly charged ions.

The comparison between the single channels (Figs. 2 and 3 ) and the coincidence channels (Figs. 4 and 5) provides some more interesting elements for discussion. To obtain a better visual insight into the fragmentation channels, including competitive processes, we report a complete list of the possible fragmentation patterns and other decay processes in Table I. The first step is the formation of the core-excited neutral state, which may directly dissociate into core-excited

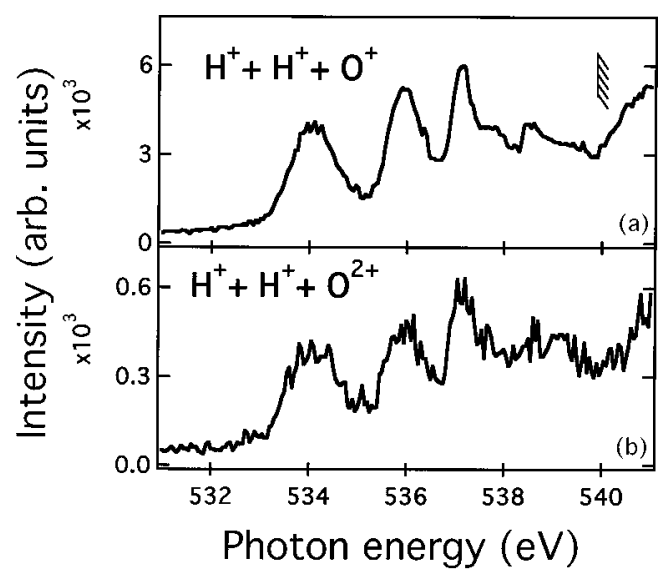

FIG. 5. Triple-coincidence channels of the dissociative ionization of $\mathrm{H}_{2} \mathrm{O}$ following $\mathrm{H}_{2} \mathrm{O}: \mathrm{O}(1 s)$ excitation and ionization: $\mathrm{H}^{+}+\mathrm{H}^{+}+\mathrm{O}^{+}$(a) and $\mathrm{H}^{+}+\mathrm{H}^{+} \mathrm{O}^{+}$(b). 
TABLE I. Possible deexcitation pathways following core excitation of water. The arrows connect a single species to its various decay products. Deexcitation processes include resonant Auger decay (e.g., $\mathrm{H}_{2} \mathrm{O}^{* *} \rightarrow \mathrm{H}_{2}\left(\mathrm{O}^{+}+1 \mathrm{e}^{-}\right.$), normal Auger decay (e.g., $\mathrm{O}^{+*} \rightarrow \mathrm{O}^{2+}+1 \mathrm{e}^{-}$), and fragmentation (e.g., $\left.\mathrm{OH}^{+*} \rightarrow \mathrm{O}^{+}+\mathrm{H}\right)$. Pathways for $\mathrm{OH}^{*}, \mathrm{OH}^{+}, \mathrm{O}^{*}$, and $\mathrm{O}^{+}$are shown only once for the sake of simplicity.

(a)

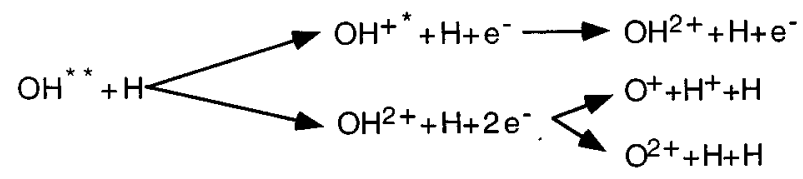

(b)

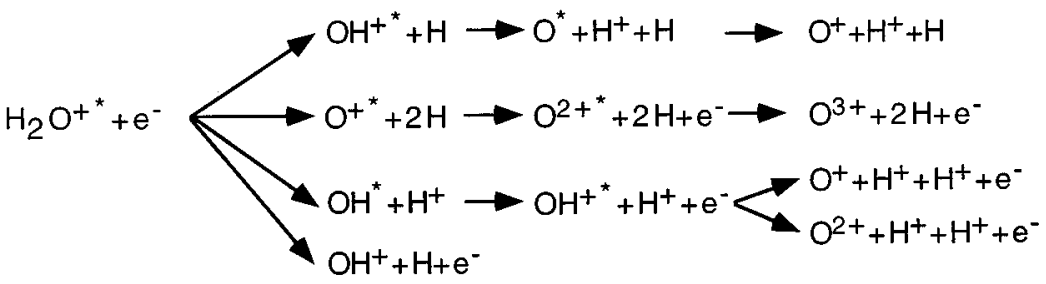

(c)

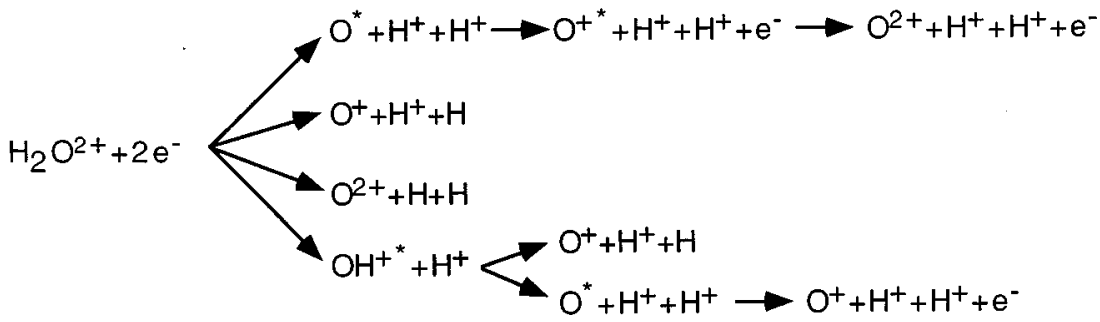

neutral $\mathrm{OH}^{* *}$ and neutral $\mathrm{H}$ fragments with a subsequent Auger decay into $\mathrm{OH}^{+*}+\mathrm{H}$ [see Table I(a)]. Such ultrafast dissociation may occur due to the strongly repulsive nature of the core-excited states, e.g., predicted in the simple $z+1$ model. The relevant time scales of neutral dissociation compared to the Auger lifetime suggest, however, a competition between both processes. Corresponding studies of inner-shell excitations in halogenides have shown that even for the decay of the $3 d$ and $2 p$ holes involved in these cases also a significant fraction of molecular Auger decay does occur [15-17]. The proof of the statement that molecular Auger decay, in the present case, occurs at comparable atomiclike strengths certainly has to be the subject of further fragmentation and electron emission studies in the core and the inner valence region. Tables I(b) and I(c) describe decays through resonant molecular Auger processes into singly charged species (one-hole states corresponding to participator decay, or two-hole-one-particle states populated by spectator decay [see Table I(b)]). The probability of fragmentation is higher for spectator transitions, since final-state potential curves are usually more repulsive than those of participator states due to the antibonding character of the corresponding two-holesone electron states. Dissociation of $\mathrm{H}_{2} \mathrm{O}^{+}$gives mainly rise to singly charged $\mathrm{H}^{+}, \mathrm{O}^{+}$, and $\mathrm{OH}^{+}$ions. Another possible process is the direct formation of doubly charged water molecules due to shake-off processes, which subsequently decay by producing more than one charged species [see Table I(c)].
First we will discuss the most intense channels $\left(\mathrm{H}^{+}, \mathrm{OH}^{+}, \mathrm{O}^{+}, \mathrm{O}^{2+}\right)$ shown in Figs. 2 and 3. We notice that the ion yields are rather similar for $\mathrm{H}^{+}$and $\mathrm{O}^{+}$, but different for $\mathrm{OH}^{+}$and $\mathrm{O}^{2+}$, where the first two peaks have different intensity ratios, the first peak being the more intense. Close examination of the single scans in Figs. 2 and 3 reveals more subtle differences. If we compare the total ion yield with the $\mathrm{H}^{+}$yield, which is the most intense single channels, we observe that the first peak is relatively weaker in the latter curve. All these differences can be attributed to the importance of the emission of a neutral fragment, namely $\mathrm{H}$ neutral. We cannot detect it, but we can qualitatively derive the relative weight of this decay fragment by comparing the single and coincidence channels. Doing this for $\mathrm{OH}^{+}$, we notice that in the coincidence channel $\mathrm{OH}^{+} / \mathrm{H}^{+}$(Fig. 4) the ratio between the first and second peak is comparable to the analogous ratio of the total yield. The difference in the intensity ratio in the $\mathrm{OH}^{+}$single channel can thus be attributed to the processes where the partner is $\mathrm{H}$ neutral and not $\mathrm{H}^{+}$. The same behavior can be observed when comparing the single scan for $\mathrm{O}^{2+}$ and the coincidence channel $\mathrm{O}^{2+} / \mathrm{H}^{+}$. Therefore, although in our experimental conditions it is not possible to detect $\mathrm{H}$ neutral, we can still derive some information on its relative weight from comparison of the $\mathrm{OH}^{+}$ and $\mathrm{H}^{+}$ion yields, both normalized to each other just below threshold, as schematically depicted in Fig. 6. The weaker first peak in the $\mathrm{H}^{+}$yield compared to the total ion yield can 


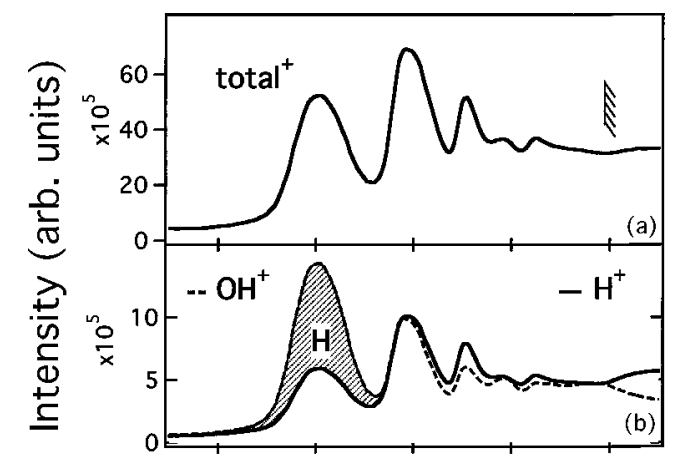

Photon energy (eV)

FIG. 6. Comparison of the $\mathrm{OH}^{+}$single-channel ionization rate with the $\mathrm{H}^{+}$single-channel ionization rate. The two rates were normalized to each other at photon energies just below the $\mathrm{O}(1 \mathrm{~s})$ threshold, where most single-ionization channels behave similar with regard to the total ionization yield.

be explained by the same reasons because all processes contribute to the total ion yield, including those which do not produce $\mathrm{H}^{+}$, and the relative weight of $\mathrm{H}$ neutral seems to be more important in correspondence to the first resonance (see the above discussion).

How can this different behavior in the $\mathrm{H}$ production yield between the first two resonances be understood? The most probable explanation lies in the energy positions of the levels involved in the excitation and decay process. The majority of final states of the spectator decay of the first resonance is still below the double-ionization threshold and, therefore, metastable. In this situation, dissociation to singly charged species with one or two neutral hydrogen atoms are allowed [see processes in Table $I(b)]$. In contrast to this, the Auger final states of the second resonance are already above the doubleionization threshold. Therefore, dissociation into either doubly charged ions or singly charged ion pairs becomes possible. This basically explains the observed behavior. The discussed situation is schematically shown in Fig. 7.

A different behavior can be observed for $\mathrm{O}^{+}$. The intensity ratio for the first two peaks in the $\mathrm{O}^{+}$single scan and in the $\mathrm{O}^{+} / \mathrm{H}^{+}$coincidence scan is almost the same. Therefore, it seems that for this channel the importance of the $\mathrm{H}$ neutral yield is minor. However, if we have a closer look at Table I, we notice that $\mathrm{O}^{+}$has an additional decay pathway which is atomic normal Auger decay. Therefore the apparent difference in the behavior of the $\mathrm{OH}^{+}$and $\mathrm{O}^{2+}$ species and the $\mathrm{O}^{+}$ ions can be explained on the ground of a competitive fast atomic Auger decay of $\mathrm{O}^{+}$leading to $\mathrm{O}^{2+}$. As a consequence, the relative strength of the channel $\mathrm{O}^{+}+\mathrm{H}+\mathrm{H}$ cannot be assessed exactly, since the atomic Auger decay takes place on the same time scale as molecular fragmentation, or even faster. The most likely behavior is the dissociation of the excited $\mathrm{H}_{2} \mathrm{O}^{+*}$ ion into $\mathrm{O}^{+*}$ and $2 \mathrm{H}$ followed by further Auger decay of the excited oxygen atom. This atomic cascade Auger channel is already open in the vicinity of the first resonance leading to comparable intensities of the first and second resonances in the $\mathrm{O}^{2+}$ channel, as seen in the upper part of Fig. 3.

The above discussion is supported by obtaining the rela-

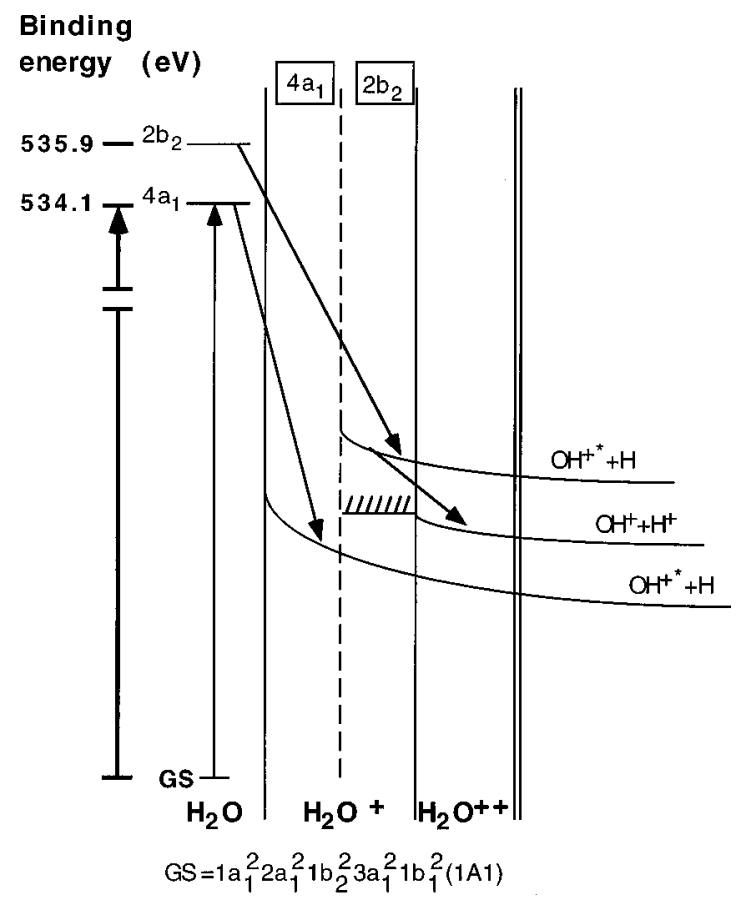

FIG. 7. Scheme of the energy potential curves involved in the decay pathways of the two lowest $\mathrm{H}_{2} \mathrm{O}: \mathrm{O}(1 s)$ excitations into $\mathrm{OH}$ fragments. The variation of the potential curves has a purely symbolic meaning, because there is no internuclear distance $r$ abscissa.

tive trends of the single channels, i.e., their branching ratios with respect to the total ion yield. In Fig. 8 we show the ratios of the $\mathrm{H}^{+}, \mathrm{OH}^{+}, \mathrm{O}^{+}$, and $\mathrm{O}^{2+}$ channels to the total ion yield. It is noticeable that the relative yield of $\mathrm{H}^{+}$shows

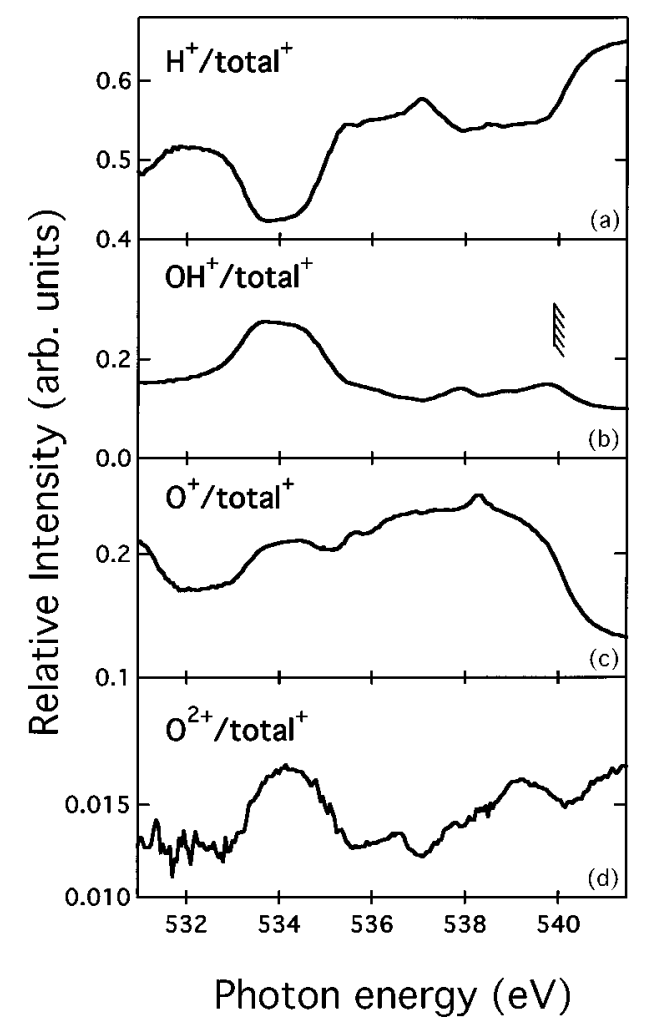

FIG. 8. Relative noncoincident ionization yields normalized to the total ionization yield. 


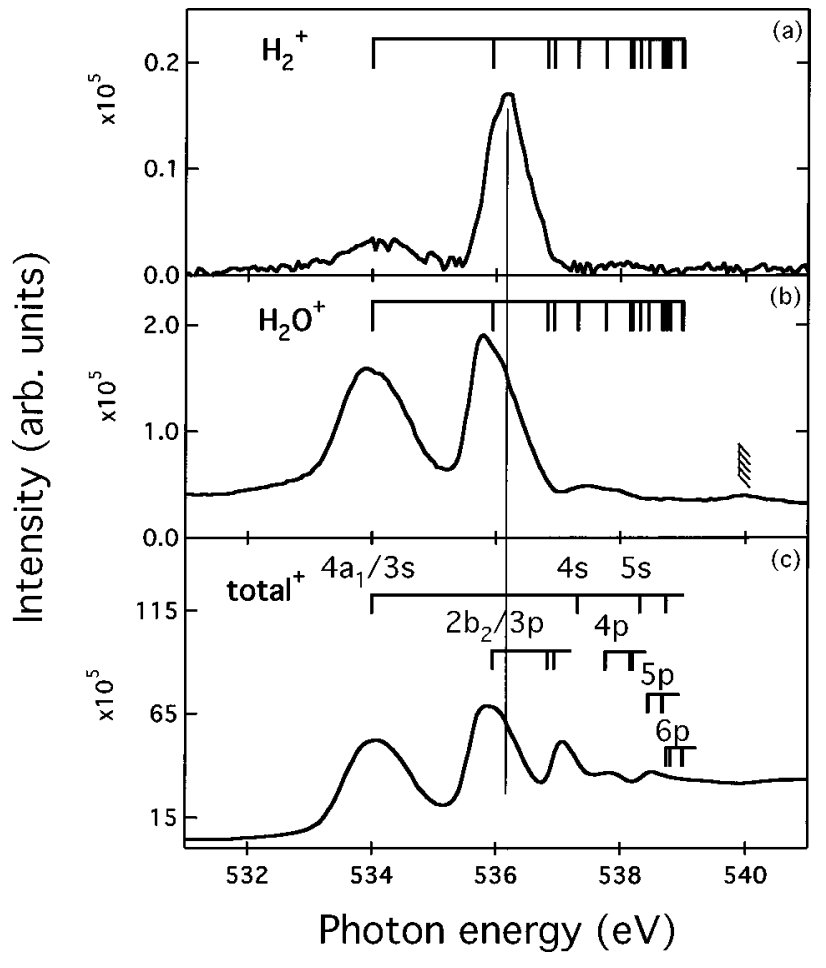

FIG. 9. Enhanced representation of the $\mathrm{H}_{2}{ }^{+}$ion yield in comparison with the $\mathrm{H}_{2} \mathrm{O}^{+}$and total ion yield. A bar diagram of all calculated excitation energies is shown above each spectrum. The position of the $\mathrm{H}_{2}{ }^{+}$peak is marked by a long vertical line.

a minimum corresponding to a transition to the first resonance, thus corroborating the idea that the $\mathrm{H}^{+}$production is less important in the vicinity of this resonance because of the competing channel $\mathrm{H}$ neutral. The inverse effect can be observed for the relative yield of $\mathrm{OH}^{+}$and $\mathrm{O}^{2+}$, where there is a maximum corresponding to a transition to the first resonance. The above-discussed different behavior of $\mathrm{O}^{+}$is confirmed by the fact that its branching ratio does not exhibit a maximum matching the behavior of $\mathrm{OH}^{+}$and $\mathrm{O}^{2+}$, but it has a rather complicated structure in the photon energy region of the first resonance.

As for the weaker channels (Figs. 2 and 3), we can observe that there is some evidence for $\mathrm{O}^{3+}$, which shows a resonant profile similar to $\mathrm{O}^{2+}$. The production of $\mathrm{O}^{3+}$ is due only to a further cascade Auger decay of $\mathrm{O}^{2+}$, and therefore the two ion yield curves mimic each other.

Some more interesting observations can be derived from the analysis of $\mathrm{H}_{2} \mathrm{O}^{+}$and $\mathrm{H}_{2}{ }^{+}$(Fig. 9). In the molecular channel, the relative intensity of molecular ions is much higher for the first two resonances. These are attributed as admixtures of empty molecular orbitals and Rydberg states. It is generally believed that fragmentation processes should be more likely to occur following transitions to empty antibonding molecular orbitals. In the present case we observe that the detection of molecular parent ions is almost zero for the photon energies corresponding to $k$-shell excitations into pure Rydberg states. This is in contrast to recent findings for other molecules, e.g., $\mathrm{CO}$, where nondissociative ionization was detected in the decay of both empty molecular orbitals and Rydberg states [5]. We also notice that we did not detect $\mathrm{H}_{2} \mathrm{O}^{2+}$, again at variance with the $\mathrm{CO}$ case, where $\mathrm{CO}^{2+}$ species were observed for all resonances below threshold [5].
This experimental finding indicates that the fragmentation of molecular ions with a charge higher than +1 is a fast process or, in other words, there are no metastable doubly charged species which may be populated by the resonant Auger decay. These two observations are consistent in the sense that the fragmentation following the decay of Rydberg excitations can be compared to the fragmentation following normal Auger decay if the excited electron is in an outermost region, and its interaction with the molecular core is negligible. Therefore, it is understandable that fragmentation following resonant Auger decay of Rydberg states gives no rise to highly excited singly charged molecular ions in the same way as normal Auger decay does not result in doubly charged ions; instead fragment ions are possibly formed in excited states.

A particularly interesting and unexpected experimental finding is the detection of $\mathrm{H}_{2}{ }^{+}$species. The formation of such species seems to be almost limited to the energy region around the $\mathrm{O} 1 s \rightarrow 2 b_{2}$ resonance. Furthermore, the peak maximum in the $\mathrm{H}_{2}{ }^{+}$yield does not correspond to the maximum in the absorption cross section, but is shifted by 450 $\mathrm{meV}$ to a higher photon energy. If we carefully observe the yield curves for some of the other species, particularly for $\mathrm{H}_{2} \mathrm{O}^{+}$and $\mathrm{OH}^{+}$, we notice that there is a shoulder corresponding to the maximum in the $\mathrm{H}_{2}{ }^{+}$yield. This structure may simply be attributed to vibrational structure, e.g., the third vibrational excitation of the symmetric bending motion with $\nu_{2}=150 \mathrm{meV}$. However, we have already pointed out that there is a general lack of vibrational structure in the yield curves, which is known to be due to the intermediate states being repulsive. A possible explanation for this may be that, after excitation to the high-photon-energy side of the $\mathrm{O}$ $1 s \rightarrow 2 b_{2}$ resonance, and before the fragmentation, the electron decay reaches molecular ions which are highly vibrationally excited. In particular, if the vibrational excitation involves the bending mode, we can imagine a highly excited motion which brings the two hydrogen atoms close together. Therefore, a possible decay path of such a vibrationally excited state is the direct formation of $\mathrm{H}_{2}^{+}$during the subsequent fragmentation; even our resolution is insufficient to resolve these particular bending mode vibrations. An alternative explanation is the presence of two levels of $b_{2}$ symmetry contributing to the resonant peak at $535.9 \mathrm{eV}$, which could correspond to an antibonding molecular orbital and to one of the $3 p$ Rydberg states. A peak asymmetry in the shape was also observed in an angle-resolved low-resolution total-ion-yield experiment, and was attributed to two almost overlapping electronic states [9]. However, the direct reaction leading to the formation of $\mathrm{a} \mathrm{H}_{2}{ }^{+}$ion seems more likely to take place because of a high excitation of the bending mode. Theoretical support would be important to confirm either interpretation.

We have previously discussed the general picture of photofragmentation as a subsequent process with respect to electron decay, and the fact that exceptions to this model are molecules which exhibit ultrafast dissociation, i.e., the nuclear motion begins on the same time scale as the electron decay. Water has been predicted to show ultrafast dissociation [7]. However, the signature for ultrafast dissociation in condensed water is the yield of only $\mathrm{H}^{+}$ions, accompanied by a symmetry-breaking effect due to the surface or to the 
hydrogen bonding in the solid phase; thus the comparison in our conditions is not straightforward.

\section{SUMMARY}

A high-resolution partial ion yield of isolated water has been obtained following below-threshold core excitations in the photon energy range of 531-542 eV, including a transition from the $\mathrm{O} 1 s$ core level to intermediate states with either a mixed molecular-orbital-Rydberg character or a pure Rydberg one. The main results are the observation of a common trend in normal Auger versus resonant Auger decay, where the former leads to the production of ion pairs and the latter to singly charged species; the assessment of the relative weight of $\mathrm{H}$ neutral, which is found to be more important following the decay of the $\mathrm{O} 1 s \rightarrow 4 a_{1}$ primary exci- tation; the presence of normal Auger decay in the atomic fragmentation, faster than nuclear motion; and the more dissociative character of the Auger final states following the decay of pure Rydberg states compared with the decay of mixed molecular-orbital-Rydberg states and the formation of $\mathrm{H}_{2}{ }^{+}$, most likely following the excitation of the symmetric bending vibration. Theoretical support would be desirable to obtain a better insight into the nature of these complex decay processes.

\section{ACKNOWLEDGMENTS}

This work was supported by the Deutsche Forschungsgemeinschaft under Contract No. Be860/14-2. One of us (M. N. P.) is indebted to the Max-Planck-Gesellschaft for financial support.
[1] I. Nenner and P. Morin, in VUV and Soft X-Ray Photoionization, edited by U. Becker and D. Shirley (Plenum, New York, 1996).

[2] See, e.g., Desorption Induced by Electronic Transitions, DIET $I-V$, Springer Series in Chemical Physics Vol. 24, and Springer Series in Surface Science Vols. 4, 13, 19, and 31 (Springer-Verlag, Berlin, 1983-1993).

[3] W. Wurth and D. Menzel, in Application of Synchrotron Radiation, edited by W. Eberhardt, Springer Series in Surface Science Vol. 35 (Springer-Verlag, Berlin, 1995), p. 171.

[4] D. L. Hansen, M. E. Arrasate, J. Cotter, G. R. Fisher, K. T. Leung, J. C. Levin, R. Martin, P. Neill, R. C. C. Perera, I. A. Sellin, M. Simon, Y. Uehara, B. Vanderford, S. B. Whitfield, and D. W. Lindle, Phys. Rev. A 57, 2608 (1998).

[5] N. Saito, F. Heiser, O. Hemmers, A. Hempelmann, K. Wieliczek, J. Viefhaus, and U. Becker, Phys. Rev. A 51, R4313 (1995).

[6] P. Erman, A. Karawajczyk, F. Rachlew-Kallne, and C. Stromholm, J. Phys. B 28, 2069 (1995).

[7] D. Coulman, A. Puschmann, U. Höfer, H.-P. Steinrück, W. Wurth, P. Feulner, and D. Menzel, J. Chem. Phys. 93, 58 (1990), and references therein.

[8] See, e.g., T. Hayaishi, A. Yagishita, K. Ito, M. Kihara, and Y.
Morioka, J. Phys. B 20, 1207 (1987); J. E. Reutt, L. S. Wang, Y. T. Lee, and D. A. Shirley, J. Chem. Phys. 85, 6928 (1986).

[9] D. Y. Kim, K. Lee, C. I. Ma, M. Mahalingam, and D. M. Hansen, J. Chem. Phys. 97, 5915 (1992).

[10] M. N. Piancastelli, B. Kempgens, U. Hergenhahn, A. Kivimäki, K. Maier, A. Rüdel, and A. M. Bradshaw, Phys. Rev. A (to be published).

[11] N. Saito, F. Heiser, O. Hemmers, K. Wieliczek, J. Viefhaus, and U. Becker, Phys. Rev. A 54, 2004 (1996).

[12] N. Saito and I. H. Suzuki, Phys. Rev. Lett. 61, 2740 (1988).

[13] J. Schirmer, A. B. Trofimov, K. J. Randall, J. Feldhaus, A. M. Bradshaw, Y. Ma, C. T. Chen, and F. Sette, Phys. Rev. A 47, 1136 (1993).

[14] A. Cesar, H. Ågren, and V. Carravetta, Phys. Rev. A 40, 187 (1989).

[15] E. Kukk, H. Aksela, O.-P. Sairanen, S. Aksela, A. Kivimäki, E. Nommiste, A. Ausmees, A. Kikas, S. J. Osborne, and S. Svensson, J. Chem. Phys. 104, 4475 (1996).

[16] A. Menzel, B. Langer, J. Viefhaus, S. B. Whitfield, and U. Becker, Chem. Phys. Lett. 258, 265 (1996).

[17] Z. F. Liu, G. M. Bancroft, K. H. Tan, and M. Schachter, Phys. Rev. A 48, R4019 (1993). 